01

\title{
Объемные нелинейные упругие волны деформации в двуслойном коаксиальном цилиндрическом стержне
}

\author{
(c) И.А. Гула, А.М. Самсонов \\ Физико-технический институт им. А.Ф. Иофрфе РАН, \\ 194021 Санкт-Петербург, Россия \\ I e-mail: samsonov@math.ioffe.ru
}

(Поступило в Редакцию 7 апреля 2017 г.)

Рассматрена задача о распространении нелинейно-упругих длинных продольных волн деформации в двуслойном коаксильном цилиндрическом стержне с идеальным контактом между слоями. Получены выражения для поперечных перемещений через продольные и удовлетворяющие с заданной точностью условию свободной границы и условию непрерывности перемещений и напряжений на поверхности раздела слоев. Показано, как выражения обобщают известные гипотезу плоских сечений и гипотезу Лява для изотропного однородного стержня. Выведено уравнение распространения продольной волны нелинейноупругой деформации и исследовано его частное решение в виде уединенной бегущей волны.

DOI: 10.21883/JTF.2017.12.45198.2295

\section{Введение}

Композитные материалы (композиты) широко используются в современной промышленности. При их исследовании в отдельный класс часто выделяют многокомпонентные материалы, которые состоят из разных однородных изотропных слоев - слоистые композиты. Изучение динамики поведения таких композитов и выявление их механических повреждений являются важными задачами, одним из перспективных методов решения которых являются неразрушающие испытания. Они позволяют контролировать целостность образцов и выявлять повреждения, основываясь на эволюции распространящихся волн деформации, а с другой стороны, изучать физические свойства материала волновода по параметрам этих волн. Особый интерес представляют нелинейные продольные волны деформации, затухание которых очень мало́ по сравнению с линейными продольными волнами, причем даже в полимерах, обладающих сильной диссипацией, см. [1]. Однако теоретические исследования распространения волн деформации в слоистых композитных волноводах пока недостаточно развиты,в частности, только недавно было показано, что в слоистых композитах, состоящих из конечного числа плоских слоев, могут распространяться объемные нелинейные волны деформации, см., например, [2-5]. Как теоретически, так и экспериментально интенсивно исследуются задачи распространения объемных нелинейных волн в плоских деламинированных волноводах $[3,6,7]$

Линейная задача о распространении продольных волн в двуслойном упругом стержне была рассмотрена в [8], очевидно, физическая нелинейность материалов и геометрическая нелинейность деформации не были учтены. Авторы использовали метод асимптотических разложений [9], сведя задачу к последовательности классических волновых уравнений.
Модель распространения нелинейных упругих волн в изотропном цилиндрическом стержне в виде уравнения с двумя дисперсиями (УДД) и ее уточнения были предложены в 80-х годах; изложению этого подхода посвящены книга [10] и цитированная там литература. Недавно в [11] была рассмотрена задача о распространении нелинейных волн деформации вдоль пластины, состоящей из периодического двухкомпонентного композита с бесконечным числом плоских слоев, причем заданная периодичность позволила свести исходную двумерную задачу к единственному одномерному нелинейному уравнению для продольных волн деформации.

Задача становится гораздо сложнее, если число нелинейно упругих слоев в композите невелико, слои не плоские, и периодичность отсутствует. Целью настоящей работы является разработка модели распространения длинных нелинейных продольных упругих волн деформации в двуслойном коаксиальном стержне с круглым сечением, которая имеет большое практическое значение. Получены асимптотически точные выражения поперечных перемещений через продольные, позволяющие удовлетворить условие свободной от нагружения внешней границы стержня и обеспечить непрерывность перемещений и напряжений на границе раздела слоев. Это позволило свести задачу к одномерному нелинейному уравнению относительно единственной неизвестной функции и получить решение в виде объемной нелинейной уединенной волны (солитона) деформации. Параметры этой волны приведены в размерном виде, пригодном для расчетов в реальном физическом эксперименте.

\section{1. Вывод уравнений модели}

Рассмотрим стержень с круглым сечением, состоящий из двух различных коаксиальных слоев. Введем 


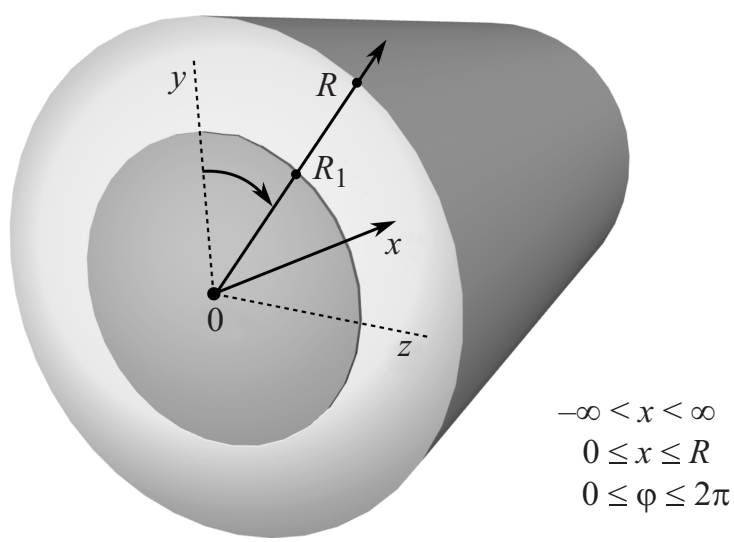

Рис. 1. Двуслойный коаксиальный стержень с круглым сечением.

цилиндрическую систему координат с началом в центре одного из торцов стержня, ось $O x$ направим вдоль продольной оси стержня, ось $O r$ - по радиальному направлению. Пусть длина стержня велика по сравнению с радиусом его внешнего слоя, равным $R$, а радиус внутреннего слоя равен $R_{1}<R$ (рис. 1). Допустим, что кручение стержня отсутствует, и задача обладает осевой симметрией, тогда введем вектор перемещений $\mathbf{U}(x, r, t)=U(x, r, t) \mathbf{i}+V(x, r, t) \mathbf{j}$, где символом $t$ обозначена временная переменная.

Функции перемещений и компоненты тензора напряжений $\mathbf{P}$ в слоях будем обозначать верхним индексом в скобках: $U^{(i)}(x, r, t), P_{r r}^{(i)}(x, r, t)$, а константы упругости - нижним индексом: $\lambda_{i}, \mu_{i}$ и т. п.

Тензор конечных деформаций Коши-Грина [10] имеет вид

$C=\frac{1}{2}\left[\begin{array}{ccc}2 \partial_{x} U+\left(\partial_{x} U\right)^{2} & \partial_{r} U+\partial_{x} U \partial_{r} U+\partial_{x} V \partial_{r} V & 0 \\ \partial_{r} U+\partial_{x} V+\partial_{x} U \partial_{r} U & 2 \partial_{r} V+\left(\partial_{r} U\right)^{2}+\left(\partial_{r} V\right)^{2} & 0 \\ 0 & 0 & 2 V / r+V^{2} / r^{2}\end{array}\right]$.

Для функции плотности потенциальной энергии каждого из материалов слоев примем модель нелинейно упругого материала Мурнагана [12]:

$$
\begin{aligned}
\Pi= & \frac{\lambda+2 \mu}{2}\left(I_{1}(\mathbf{C})\right)^{2}-2 \mu I_{2}(\mathbf{C})+\frac{l+2 m}{3}\left(I_{1}(\mathbf{C})\right)^{3} \\
& -2 m I_{1}(\mathbf{C}) I_{2}(\mathbf{C})+n I_{3}(\mathbf{C})
\end{aligned}
$$

где $\lambda, \mu$ - постоянные Лямэ, $l, m, n-$ модули Мурнагана, $I_{1}, I_{2}, I_{3}$ - инварианты тензора деформаций $\mathbf{C}$.

При нелинейных деформациях результат приложения сил (напряжений) зависит от пути деформирования, поэтому при постановке задачи в отсчетных координатах (до деформирования) используется тензор ПиолыКирхгоффа $\mathbf{P}=\partial П / \partial \mathbf{C}(\mathbf{E}+\nabla \mathbf{U})$, определенный в отсчетной конфигурации. Запишем его компоненты $P_{r r}$, $P_{r x}$ до слагаемых второго порядка по степеням компо- нент тензора $\nabla \mathbf{U}$ включительно:

$$
\begin{aligned}
P_{r r} & =\lambda \partial_{x} U+\lambda \frac{V}{r}+(\lambda+2 \mu) \partial_{r} V+\frac{\lambda+2 l}{2} \frac{V^{2}}{r^{2}} \\
& +\frac{\lambda+2 \mu+m}{2}\left(\partial_{r} U\right)^{2}+(2 l-2 m+n) \partial_{x} U \frac{V}{r} \\
& +\frac{\lambda+2 l}{2}\left(\partial_{x} U\right)^{2}+(\lambda+2 l) \frac{V}{r} \partial_{r} V \\
& \left.+(\lambda+2 l) \partial_{x} U \partial_{r} V+\frac{3 \lambda+6 \mu+2 l+4 m}{2}\right]\left(\partial_{r} V\right)^{2} \\
& +(\mu+m) \partial_{r} U \partial_{x} V+\frac{\lambda+2 \mu+m}{2}\left(\partial_{x} V\right)^{2} \\
P_{r x} & =\mu \partial_{r} U+\mu \partial_{x} V+\frac{2 \lambda+2 m-n}{2} \partial_{r} U \frac{V}{r} \\
& +(\lambda+2 \mu+m) \partial_{x} U \partial_{r} U+(\lambda+2 \mu+m) \partial_{r} U \partial_{r} V \\
& +\frac{2 m-n}{2} \frac{V}{r} \partial_{x} V+(\mu+m) \partial_{x} U \partial_{x} V+(\mu+m) \partial_{x} V \partial_{r} V
\end{aligned}
$$

Введем масштабы для $x, r$ и $t$ в виде $\Lambda, R$ и $\Lambda / c_{0}$ соответственно, где $\Lambda$ - характерная длина продольных волн в стержне, $c_{0}$ - характерная скорость распространения линейных волн, вообще говоря, отличная от „стержневой“ скорости. Положим, что масштабы компонент деформаций $U_{x}$ и $V_{r}$ одинаковы и равны $A$, тогда масштабы для перемещений $U$ и $V$ можно записать соответственно в виде $\Lambda A$ и $R A$. Введем безразмерные переменные $x^{\prime}=x / \Lambda, r^{\prime}=r / R, t^{\prime}=t \Lambda / c_{0}$, безразмерные функции $U^{\prime}=U /(\Lambda A), V^{\prime}=V /(R A)$ и безразмерный радиус внутреннего стержня $\alpha=R_{1} / R$. Положим, что деформации малы, $A \ll 1$, будем рассматривать длинные продольные волны, $R / \Lambda \ll 1$, и по аналогии c [10] постулируем соотношение $A=R^{2} / \Lambda^{2}$, после чего можно ввести малый параметр $\varepsilon^{2}=A=R^{2} / \Lambda^{2}$.

Запишем компоненты $P_{r r}, P_{r x}$ тензора ПиолыКирхгоффа в безразмерных переменных и соберем слагаемые при одинаковых степенях малого параметра (штрихи опущены):

$$
\begin{aligned}
& P_{r r}=\varepsilon^{2}\left(\lambda \partial_{x} U+\lambda \frac{V}{r}+(\lambda+2 \mu) \partial_{r} V+\frac{\lambda+2 \mu+m}{2}\left(\partial_{r} U\right)^{2}\right) \\
& +\varepsilon^{4}\left(\frac{\lambda+2 l}{2} \frac{V^{2}}{r^{2}}+(2 l-2 m+n) \partial_{x} U \frac{V}{r}+\frac{\lambda+2 l}{2}\left(\partial_{x} U\right)^{2}\right. \\
& +(\lambda+2 l) \frac{V}{r} \partial_{r} V+(\lambda+2 l) \partial_{x} U \partial_{r} V \\
& \left.+\frac{3 \lambda+6 \mu+2 l+4 m}{2}\left(\partial_{r} V\right)^{2}+(\mu+m) \partial_{r} U \partial_{x} V\right) \\
& +\varepsilon^{6} \frac{\lambda+2 \mu+m}{2}\left(\partial_{x} V\right)^{2},
\end{aligned}
$$




$$
\begin{aligned}
& P_{r x}=\varepsilon \mu \partial_{r} U \\
& +\varepsilon^{3}\left(\mu \partial_{x} V+\frac{2 \lambda+2 m-n}{2} \partial_{r} U \frac{V}{r}\right. \\
& \left.+(\lambda+2 \mu+m) \partial_{x} U \partial_{r} U+(\lambda+2 \mu+m) \partial_{r} U \partial_{r} V\right) \\
& +\varepsilon^{5}\left(\frac{2 m-n}{2} \frac{V}{r} \partial_{x} V+(\mu+m) \partial_{x} U \partial_{x} V+(\mu+m) \partial_{x} V \partial_{r} V\right)
\end{aligned}
$$

Теперь разложим функции перемещений в ряд по четным степеням малого параметра $\varepsilon$ :

$$
\begin{aligned}
& U(x, r, t)=U_{0}(x, r, t)+\varepsilon^{2} U_{2}(x, r, t)+\ldots, \\
& V(x, r, t)=V_{0}(x, r, t)+\varepsilon^{2} V_{2}(x, r, t)+\ldots,
\end{aligned}
$$

и убедимся в том, что разложения (7), (8) сохраняют вид разложений $P_{r r}, P_{r x}$ в ряды по $\varepsilon$ и не добавляют новых слагаемых

$$
\begin{aligned}
& P_{r r}(x, r, t)=\varepsilon^{2} P_{2, r r}(x, r, t)+\varepsilon^{4} P_{4, r r}(x, r, t)+\ldots, \\
& P_{r x}(x, r, t)=\varepsilon P_{1, r r}(x, r, t)+\varepsilon^{3} P_{3, r x}(x, r, t)+\ldots
\end{aligned}
$$

Подставляя (7-10) в (5),(6) и собирая слагаемые при соответствующих степенях малого параметра, в главном порядке по $\varepsilon$ в $P_{r x}$ (здесь и далее будут использоваться индексы, соответствующие слоям стержня) имеем

$$
P_{1, r x}^{(i)}=\mu \partial_{r} U_{0}^{(i)}
$$

Функции $U_{0}^{(i)}$ будем искать в таком виде, чтобы выполнялись условия

$\left.U_{0}^{(1)}\right|_{r=\alpha}=\left.U_{0}^{(2)}\right|_{r=\alpha},\left.\quad P_{1, r x}^{(1)}\right|_{r=\alpha}=\left.P_{1, r x}^{(2)}\right|_{r=\alpha},\left.P_{1, r x}^{(2)}\right|_{r=1}=0$,

откуда получаем $U_{0}^{(1)}=U_{0}^{(2)}=F(x, t)$. Таким образом, доказано, что в задаче о двуслойном стержне, как и в задаче об изотропном стержне [10], гипотезы плоских сечений достаточно ля того, чтобы занулить касательные напряжения на внешней границе стержня в главном порядке $\varepsilon$. Кроме того, удовлетворены условия непрерывности перемещений и напряжений на поверхности раздела слоев. Аналогичные выражения для $U_{0}^{(i)}$ были получены при рассмотрении линейной задачи о двуслойном цилиндрическом стержне [8].

Учитывая выражения для $U_{0}^{(i)}$, имеем для $P_{2, r r}^{(i)}$ :

$$
P_{2, r r}^{(i)}=\lambda_{i} \frac{V_{0}^{(i)}}{r}+\lambda_{i} \partial_{x} F+\left(\lambda_{i}+2 \mu_{i}\right) \partial_{r} V_{0}^{(i)}
$$

Для нахождения $V_{0}^{(i)}$ удовлетворяем условия

$\left.V_{0}^{(1)}\right|_{r=\alpha}=\left.V_{0}^{(2)}\right|_{r=\alpha},\left.P_{2, r r}^{(1)}\right|_{r=\alpha}=\left.P_{2, r r}^{(2)}\right|_{r=\alpha},\left.P_{2, r r}^{(2)}\right|_{r=1}=0$,

откуда находим

$$
V_{0}^{(1)}=-\frac{a_{0}}{d_{0}} r \partial_{x} F, V_{0}^{(2)}=-\left(\frac{a_{0}-\sigma_{0}}{d_{0}} r+\frac{\sigma_{0}}{d_{0}} \frac{\alpha^{2}}{r}\right) \partial_{x} F,
$$

где

$$
\begin{gathered}
a_{0}=\frac{1}{2}\left(\frac{\lambda_{1}}{\lambda_{2}}\left(\frac{\lambda_{2}}{\mu_{2}}+\alpha^{2}\right)+\frac{\lambda_{1}}{\lambda_{2}}+\left(1-\alpha^{2}\right)\right), \\
d_{0}=2 a_{0}+\frac{\mu_{1}}{\mu_{2}}+\frac{\left(1-\alpha^{2}\right) \mu_{1}+\left(1-\alpha^{2}\right) \mu_{2}}{\lambda_{2}}, \\
\sigma_{0}=\frac{1}{2}\left(\frac{\lambda_{1}}{\lambda_{2}}-\frac{\mu_{1}}{\mu_{2}}\right) .
\end{gathered}
$$

Аналогичный результат был получен для перемещений $V_{0}^{(i)}$ в линейной задаче [8]. В выражении для $V_{0}^{(2)}$ присутствует слагаемое, сингулярное по $r$ при $r \rightarrow 0$, однако выражение для $V_{0}^{(2)}$ справедливо лишь для $r \in(a ; 1]$, вследствие чего сингулярность не возникает.

С учетом найденных $U_{0}^{(i)}$ и $V_{0}^{(i)}$ имеем для $P_{3, r x}^{(i)}$ :

$$
\begin{gathered}
P_{3, r x}^{(1)}=\mu_{1} \partial_{r} U_{2}^{(1)}-\frac{\mu_{1} a_{0}}{d_{0}} r \partial_{x x} F \\
P_{2, r x}^{(2)}=\mu_{2} \partial_{r} U_{2}^{(2)}-\left(\frac{a_{0}-\sigma_{0}}{d_{0}} r+\frac{\sigma_{0}}{d_{0}} \frac{\alpha^{2}}{r}\right) \mu_{2} \partial_{x x} F .
\end{gathered}
$$

Функции $U_{2}^{(i)}$ теперь должны удовлетворять условиям $\left.U_{2}^{(1)}\right|_{r=\alpha}=\left.U_{2}^{(2)}\right|_{r=\alpha},\left.P_{3, r x}^{(1)}\right|_{r=\alpha}=\left.P_{3, r x}^{(2)}\right|_{r=\alpha},\left.P_{3, r x}^{(2)}\right|_{r=1}=0$, откуда находим выражения

$$
\begin{gathered}
U_{2}^{(1)}=a_{1} \frac{r^{2}}{2} \partial_{x x} F, \\
U_{2}^{(2)}=\left(a_{1}-\sigma_{1} \frac{2 \ln \alpha}{\alpha^{2}}\right) \frac{r^{2}}{2} \partial_{x x} F+\sigma_{1} \ln r \partial_{x x} F,
\end{gathered}
$$

где введены коэффициенты

$$
\begin{gathered}
\alpha=\frac{a_{0}}{d_{0}}-\frac{\sigma_{0}}{d_{0}} \frac{\left(1-\alpha^{2}\right)(1-2 \ln \alpha)}{\left(\alpha^{2}-2 \ln \alpha\right) \frac{\mu_{1}}{\mu_{2}}+\left(1-\alpha^{2}\right)}, \\
\sigma_{1}=\frac{\sigma_{0}}{d_{0}} \frac{\alpha^{2}\left(1-\alpha^{2}\right)\left(1-\frac{\mu_{1}}{\mu_{2}}\right)}{\left(\alpha^{2}-2 \ln \alpha\right) \frac{\mu_{1}}{\mu_{2}}+\left(1-\alpha^{2}\right)} .
\end{gathered}
$$

Учитывая выражения для $U_{0}^{(i)}, V_{0}^{(i)}$, для $P_{4, r r}^{(i)}$ получим

$$
\begin{aligned}
P_{4, r r}^{(1)} & =2 \mu_{1} \partial_{r} V_{2}^{(1)}+\lambda_{1}\left(\frac{V_{2}^{(1)}}{r}+\partial_{r} V_{2}^{(1)}\right)+\lambda_{1} a_{1} \frac{r^{2}}{2} \partial_{x x x} F \\
& +\left(\frac{\lambda_{1}+2 l_{1}}{2}-\frac{a_{0}}{d_{0}}\left(4 l_{1}-2 m_{1}+n_{1}+\lambda_{1}\right)\right. \\
& \left.\left.+\frac{a_{0}^{2}}{d_{0}^{2}}\left(4 l_{1}+2 m_{1}+3\left(\lambda_{1}+\mu_{1}\right)\right)\right)\right)\left(\partial_{x} F\right)^{2},
\end{aligned}
$$

Журнал технической физики, 2017, том 87, вып. 12 


$$
\begin{aligned}
P_{4, r r}^{(1)} & =2 \mu_{2} \partial_{r} V_{2}^{(2)}+\lambda_{2}\left(\frac{V_{2}^{(2)}}{r}+\partial_{r} V_{2}^{(2)}\right) \\
& +\lambda_{2}\left(a_{1} \frac{r^{2}}{2}+\sigma_{1}\left(\ln r-\frac{r^{2}}{\alpha^{2}} \ln \alpha\right)\right) \partial_{x x x} F \\
& +\left(\frac{\lambda_{2}+2 l_{2}}{2}-\frac{a_{0}}{d_{0}}\left(4 l_{2}-2 m_{2}+n_{2}+\lambda_{2}\right)\right. \\
& +\frac{a_{0}^{2}}{d_{0}^{2}}\left(4 l_{2}+2 m_{2}+3\left(\lambda_{2}+\mu_{2}\right)\right) \\
& +\frac{\sigma_{0}}{d_{0}}\left(4 l_{2}-\left(1-\frac{\alpha^{2}}{r^{2}}\right)\left(2 m_{2}-n_{2}\right)+\left(1+\frac{\alpha^{2}}{r^{2}}\right) \lambda_{2}\right) \\
& +\frac{\sigma_{0}^{2}}{d_{0^{2}}}\left(2 \lambda_{2}+4 l_{2}+\left(1+\frac{\alpha^{2}}{r^{2}}\right)^{2}\left(\lambda_{2}+2 m_{2}+3 \mu_{2}\right)\right) \\
& -\frac{2 a_{0} \sigma_{0}}{d_{0}^{2}}\left(2 \lambda_{2}+4 l_{2}+\left(1+\frac{\alpha^{2}}{r^{2}}\right)\right. \\
& \left.\left.\times\left(\lambda_{2}+2 m_{2}+3 \mu_{2}\right)\right)\right)\left(\partial_{x} F\right)^{2} .
\end{aligned}
$$

Для отыскания $V_{2}^{(1)}$ выберем анзац $\delta_{1} r\left(\partial_{x} F\right)^{2}+$ $=\gamma_{1} r^{3} \partial_{x x x} F$, а $V_{2}^{(2)}$ будем отыскивать в виде

$$
\left(\delta_{2} r+\frac{\eta_{2}}{r}+\frac{\chi_{2}}{r^{3}}\right)\left(\partial_{x} F\right)^{2}+\left(\gamma_{2} r^{3}+\beta_{2}(r \ln r-r)\right) \partial_{x x x} F
$$

где $\delta_{1,2}, \gamma_{1,2}, \eta_{1,2}, \chi_{1,2}, \beta_{1,2}-$ неизвестные константы, для нахождения которых имеются 3 условия

$$
\left.V_{2}^{(1)}\right|_{r=\alpha}=\left.V_{2}^{(2)}\right|_{r=\alpha},\left.P_{4, r r}^{(1)}\right|_{r=\alpha}=\left.P_{4, r r}^{(2)}\right|_{r=\alpha},\left.P_{4, r r}^{(2)}\right|_{r=1}=0 .
$$

Представим $V_{2}^{(i)}$ в виде суперпозиции линейной и нелинейной волн $V_{2, L I N}^{(i)}+V_{2, N L}^{(i)}$, где $V_{2, L I N}^{(i)}-$ линеиные по $F(x, t)$ функции, т. е. содержащие только $\partial_{x x x} F$, а $V_{2, N L}^{(i)}-$ нелинейные, включают только $\left(\partial_{x} F\right)^{2}$. Функции $V_{2, \text { LIN }}^{(i)}$ содержат 3 неизвестные константы: $\gamma_{1}, \gamma_{2}$ и $\beta_{2}$, которые с помощью имеющихся условий (14) определяются однозначно. Получим

$$
\begin{aligned}
& V_{2, L I N}^{(1)}=\frac{a_{2}}{d_{2}} r^{3} \partial_{x x x} F, \\
& V_{2, L I N}^{(2)}=\frac{b_{2}}{d_{2}} r^{3} \partial_{x x x} F+\frac{a_{2}}{d_{2}} r(\ln r-1) \partial_{x x x} F,
\end{aligned}
$$

где

$$
\begin{aligned}
a_{2} & =\frac{a_{1}}{4}\left(\frac{\lambda_{1}-\lambda_{2}}{\lambda_{2}+2 \mu_{2}}\left(1-\alpha^{2}-\ln \alpha\right)+3 \frac{\lambda_{1}}{\lambda_{2}}(1-\ln \alpha)+\ln \alpha\right) \\
& -\sigma_{1} \frac{\ln \alpha(3-2 \ln \alpha)}{2 \alpha^{2}}, \\
d_{2} & =2\left(\frac{\lambda_{1}-\lambda_{2}}{\lambda_{2}+2 \mu_{2}}\left(1-\alpha^{2}-\ln \alpha\right)+3 \frac{\lambda_{1}}{\lambda_{2}}(1-\ln \alpha)+\ln \alpha\right) \\
+ & 3\left(\frac{\mu_{1}-\mu_{2}}{\lambda_{2}+2 \mu_{2}}\left(1-\alpha^{2}-\ln \alpha\right)+3 \frac{\mu_{1}}{\lambda_{2}}(1-\ln \alpha)+\frac{\mu_{2}}{\lambda_{2}} \ln \alpha\right),
\end{aligned}
$$

$$
\begin{gathered}
b_{2}=\frac{a_{1}}{4}\left(\frac{\lambda_{1}-\lambda_{2}}{\lambda_{2}+2 \mu_{2}}\left(1-\alpha^{2}-\ln \alpha\right)\right. \\
\left.+3 \frac{\lambda_{1}+2 \mu_{1}}{\lambda_{2}+2 \mu_{2}}(1-\ln \alpha)+\ln \alpha\right) \\
-\sigma_{1} \frac{\ln \alpha}{2 \alpha^{2}}\left(\frac{\lambda_{1}-\lambda_{2}}{\lambda_{2}+2 \mu_{2}}(1-\ln \alpha)\right. \\
\left.+3 \frac{\lambda_{1}+2 \mu_{1}}{\lambda_{2}+2 \mu_{2}}(1-\ln \alpha)+\ln \alpha\right) \\
c_{2}=3 \alpha^{2} a_{1} \sigma_{0} \frac{\mu_{2}}{\lambda_{2}+2 \mu_{2}}+\sigma_{1} \ln \alpha \frac{2\left(\lambda_{1}-\lambda_{2}\right)+3\left(\mu_{1}-\mu_{2}\right)}{\lambda_{2}+2 \mu_{2}} .
\end{gathered}
$$

В частном случае, при равенстве коэффициентов Лямэ $\lambda_{1}=\lambda_{2}=\lambda$ и $\mu_{1}=\mu_{2}=\mu$ у материалов слоев в (12) зануляется $\sigma_{0}$, а отношение $a_{0} / d_{0}$ становится равным $v$. Таким образом для обоих слоев выражение для $V_{0}^{(i)}$ сводится к известной гипотезе Лява. В то же время в (13) коэффициент $\sigma_{1}$ становится равным 0, и для обоих слоев получится уточнение гипотезы плоских сечений, а в (15) зануляется коэффициент $c_{2}$, и оба отношения $a_{2} / d_{2}, b_{2} / d_{2}$ сводятся к $v /(2(2 v-3))$. Следовательно, мы показали, что в случае равенства упругих модулей 2-го порядка полученные гипотезы (12), (13), (15) сводятся к уточненным выражениям, полученным в [10] при рассмотрении задачи об изотропном цилиндрическом стержне.

Функции $V_{2, N L}^{(i)}$ содержат 4 константы $\delta_{1}, \delta_{2}, \eta_{2}$ и $\chi_{2}$ и трех имеющихся условий (14) недостаточно для их определения. Можно было бы воспользоваться условиями непрерывности $P_{4, x x}^{(i)}$ и $P_{4, \varphi \varphi}^{(i)}$. Однако одновременное их использование переопределяет систему уравнений для отыскания констант, а выбор какого-либо одного из двух не очевиден. Нелинейные части выражений для $P_{4, x x}^{(i)}$ и $P_{4, \varphi \varphi}^{(i)}$ с учетом найденных $U_{0}^{(i)}, U_{2}^{(i)}$ и $V_{0}^{(i)}$ важны при расчетах, но громоздки, и потому вынесены в Приложение 1 .

На примере задачи об изотропном цилиндрическом стержне можно убедиться в том, что в двух главных порядках малого параметра е безразмерного уравнения Эйлера-Лагранжа не будет никакого вклада от $V_{2, N L}^{(i)}$ Таким образом, найденных $V_{2, N L}^{(i)}$ достаточно для вывода уравнения модели, и разложения функций перемещений в двуслойном цилиндрическом стержне в ряд по малому параметру $\varepsilon$ можно использовать в следующем виде:

$$
\begin{gathered}
U^{(1)}=F(x, t)+\varepsilon^{2} \alpha_{1} \frac{r^{2}}{2} \partial_{x x} F, r \in(0 ; \alpha] \\
U^{(1)}=F(x, t)+\varepsilon^{2}\left[\left(\alpha_{1}-\sigma_{1} \frac{2 \ln \alpha}{\alpha^{2}}\right) \frac{r^{2}}{2}+\sigma_{1} \ln r\right] \partial_{x x} F, \\
r \in(\alpha ; 1] \\
V^{(1)}=f \frac{a_{0}}{d_{0}} r \partial_{x} F+\varepsilon^{2}\left[-\frac{a_{2}}{d_{2}} r^{3} \partial_{x x x} F\right], r \in(0 ; \alpha]
\end{gathered}
$$


Таблица 1. Упругие модули ПС и ПММА [13], [14]

\begin{tabular}{|c|c|c|c|c|c|c|c|c|}
\hline & \multirow{2}{*}{\begin{tabular}{|l|} 
Плотность \\
$\rho, \mathrm{kg} / \mathrm{mm}^{3}$ \\
\end{tabular}} & \multicolumn{2}{|c|}{$\begin{array}{c}\text { Коэффициенты } \\
\text { Ляме }\end{array}$} & \multirow{2}{*}{\begin{tabular}{|c|}
$\begin{array}{c}\text { Модуль } \\
\text { Юнга }\end{array}$ \\
$E, \mathrm{~N} / \mathrm{m}^{2}, 10^{10}$ \\
\end{tabular}} & \multirow{2}{*}{\begin{tabular}{|c|}
$\begin{array}{c}\text { Коэффициент } \\
\text { Пуассона }\end{array}$ \\
$v$ \\
\end{tabular}} & \multicolumn{3}{|c|}{$\begin{array}{c}\text { Модули Мурнагана } \\
\text { 3-го порядка }\end{array}$} \\
\hline & & $\lambda, \mathrm{N} / \mathrm{m}^{2}, 10^{10}$ & $\mu, \mathrm{N} / \mathrm{m}^{2}, 10^{10}$ & & & $l, \mathrm{~W} / \mathrm{m}^{2}, 10^{10}$ & $m, \mathrm{~W} / \mathrm{m}^{2}, 10^{10}$ & $n, \mathrm{~W} / \mathrm{m}^{2}, 10^{10}$ \\
\hline ПС & 1060 & $\begin{array}{c}0.289 \\
\pm 0.001\end{array}$ & $\begin{array}{c}0.138 \\
\pm 0.001\end{array}$ & $\begin{array}{c}0.369 \\
\pm 0.003\end{array}$ & $\begin{array}{c}0.338 \\
\pm 0.001\end{array}$ & $\begin{array}{l}-1.89 \\
\pm 0.32\end{array}$ & $\begin{array}{l}-1.33 \\
\pm 0.29\end{array}$ & $\begin{array}{l}-1.00 \\
\pm 0.14\end{array}$ \\
\hline ПММА & 1160 & 0.39 & 0.186 & 0.498 & 0.339 & -1.09 & -0.77 & -0.14 \\
\hline
\end{tabular}

Таблица 2. Результаты расчетов

\begin{tabular}{|c|c|c|c|c|}
\hline & $\begin{array}{c}\text { Скорость } \\
\text { линейных } \\
\text { волн }\end{array}$ & $\mid \begin{array}{c}\text { Скорость } \\
\text { нелинейных } \\
\text { уединенных } \\
\text { волн }\end{array}$ & $\begin{array}{c}\text { Коэффицент } \\
\text { при нелинейном } \\
\text { слагаемом }\end{array}$ & $\begin{array}{c}\text { Ширина } \\
\text { солитона } \\
\text { на половине } \\
\text { амплитуды }\end{array}$ \\
\hline & $c_{0}, \mathrm{~m} / \mathrm{s}$ & $v, \mathrm{~m} / \mathrm{s}$ & $\beta, \mathrm{W} / \mathrm{m}^{2}, 10^{10}$ & FWHM, cm \\
\hline ПС & $\begin{array}{c}1866.8 \\
\pm 7.6\end{array}$ & $\begin{array}{c}1867.4 \\
\pm 7.7\end{array}$ & $\begin{array}{l}-2.786 \\
\pm 0.799\end{array}$ & $\begin{array}{l}16.9 \\
\pm 3.2\end{array}$ \\
\hline ПММА & 2071 & 2072 & -0.458 & 48.2 \\
\hline $\begin{array}{c}\text { ПС } \\
+\Pi \mathrm{MMA}\end{array}$ & 1977 & 1978 & -1.622 & 25.9 \\
\hline
\end{tabular}

$$
\begin{aligned}
& V^{(2)}=-\left(\frac{a_{0}-\sigma_{0}}{d_{0}} r+\frac{\sigma_{0}}{d_{0}} \frac{\alpha^{2}}{r}\right) \partial_{x} F \\
& +\varepsilon^{2}\left[-\left(\frac{b_{2}}{d_{2}} r^{3}+\frac{c_{2}}{d_{2}} r(1-\ln r)\right) \partial_{x x x} F\right], r \in(\alpha ; 1] .
\end{aligned}
$$

Представление (16) в размерных переменных важно для расчетов в физических экспериментах и приведено в Приложении 2.

Применение принципа Гамильтона с учетом (16) после интегрирования безразмерной двумерной плотности лагранжиана $\mathscr{L}(,$,$) по r \in(0 ; 1)$ приводит к нелинейному уравнению с двумя дисперсиями (УДД), описывающему распространение волн нелинейно-упругих продольных деформаций в двуслойном стержне:

$$
u_{t t}-u_{x x}=\varepsilon^{2}\left[\frac{B_{1}}{2 B_{0}}\left(u^{2}\right)_{x x}+\frac{c_{0}^{2} B_{2}}{B_{0}} u_{x x t t}+\frac{B_{3}}{B_{0}} u_{x x x x}\right],
$$

где $u(x, t)=F_{x}(x, t)$. В (17) „стержневая“ скорость определяется выражением $c_{0}^{2}=B_{0} / \bar{\rho}$ где $\bar{\rho}=\alpha^{2} \rho_{1}+$ $+\left(1-\alpha^{2}\right) \rho_{2}$, а „эффективный“ модуль Юнга равен $B_{0}$. Размерный вид (17), необходимый для проверки полученных результатов в физическом эксперименте, представлен в Приложении 3, там же приведены точные, но довольно громоздкие выражения для коэффициентов $B_{i}$, $i \in[0 ; 3]$.

Особый интерес представляет частный случай равенства коэффициентов Пуассона материалов слоев $v_{1}=v_{2}=v$, часто возникающий в динамике нанокомпозитных волноводов, в котором $B_{i}, i \in[0 ; 3]$ заметно упрощаются:

$$
\begin{gathered}
B_{0}=\alpha^{2} E_{1}+\left(1-\alpha^{2}\right) E_{2}, B_{1}=\alpha^{2} \beta_{1}+\left(1-\alpha^{2}\right) \beta_{2} \\
B_{2}=\frac{v(1-v)}{2}\left(\alpha^{4} \rho_{1}+\left(1-\alpha^{4}\right) \rho_{2}\right), \\
B_{3}=\frac{v}{2}\left(\alpha^{4} E_{1}+\left(1-\alpha^{4}\right) E_{2}\right),
\end{gathered}
$$

где $\beta_{i}=3 E_{i}+2 l_{i}(1-2 v)^{3}+4 m_{i}(1+v)^{2}(1-2 v)+6 n_{i} v^{2}$. В этом случае скорость распространения линейных волн $c_{0}$ достаточно просто выражается через физические постоянные материалов слоев

$$
c_{0}^{2}=\frac{\alpha^{2} E_{1}+\left(1-\alpha^{2}\right) E_{2}}{\alpha^{2} \rho_{1}+\left(1-\alpha^{2}\right) \rho_{2}} .
$$

Среди решений УДД (17), приведенного к размерному виду (см. (23) в Приложении 3), с постоянными коэффициентами в упрощенной форме (18) существует однопараметрическое решение в виде уединенной колоколообразной волны (солитона) сжатия [10], распростра-

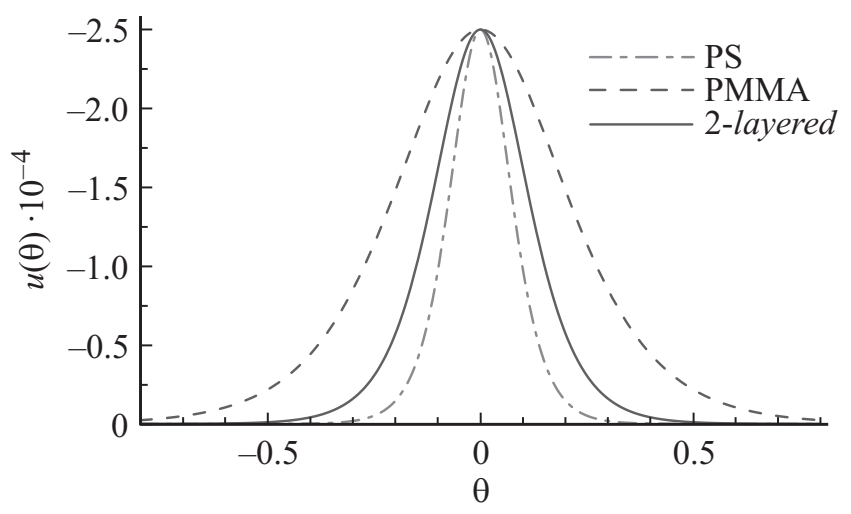

Рис. 2. Профиль уединенной волны деформации сжатия, описываемой (20) (сплошная линия), которая распространяется в двуслойном цилиндрическом волноводе радиуса $R=5 \mathrm{~mm}$. Внутренний стержень из PS имеет радиус $R_{1}=3.54 \mathrm{~mm}$, внешняя оболочка из РММА. По оси абсцисс бегущая переменная $\theta=x \pm v t$. Для сравнения на графике также представлены профили уединенных волн деформации в однородных изотропных цилиндрических стержнях из PS (штрихпунктирная линия) и РММА (штриховая линия), радиусы которых $R=5 \mathrm{~mm}$. 
няющейся со скоростью $v$ :

$$
\begin{gathered}
u(\theta)=\cosh ^{-2}\left[\theta \sqrt{\left.\frac{A \bar{\beta}}{6 v R^{2}\left(\tilde{E}-(1-v) \frac{\tilde{\rho}}{\rho}\left(\frac{A \bar{\beta}}{3}+\bar{E}\right)\right)}\right]}\right] \\
\theta=x \pm v t,
\end{gathered}
$$

где $A=3\left(\bar{\rho} v^{2}-\bar{E}\right) / \bar{\beta}<0-$ амплитуда волны, $\bar{E}=$ $=\alpha^{2} E_{1}+\left(1-\alpha^{2}\right) E_{2}, \bar{\beta}=a^{2} \beta_{1}+\left(1-\alpha^{2}\right) \beta_{2}, \tilde{E}=\alpha^{4} E_{1}+$ $+\left(1-\alpha^{4}\right) E_{2}, \tilde{\rho}=\alpha^{4} \rho_{1}+\left(1-\alpha^{4}\right) \rho_{2}$. Считая параметром амплитуду деформации, скорость солитона нетрудно выразить через упругие постоянные и плотности материалов слоев

$$
v^{2}=\frac{\frac{A}{3}\left(\alpha^{2} \beta_{1}+\left(1-\alpha^{2}\right) \beta_{2}\right)+\left(\alpha^{2} E_{1}+\left(1-\alpha^{2}\right) E_{2}\right)}{\alpha^{2} \rho_{1}+\left(1-\alpha^{2}\right) \rho_{2}},
$$

и в силу того, что $A \bar{\beta}>0$, эта скорость превосходит стержневую скорость $c_{0}$. Теперь видно, что, поскольку $\alpha \in(0 ; 1)$, то и скорость распространения линейных волн $c_{0}$, и скорость распространения солитонов $v$, и их ширина на половине высоты (FWHM - Full Width on Half Maximum) всегда будут лежать в промежутках между соответствующими величинами в каждом из однородных изотропных стержней, изготовленных из материала того или другого слоя.

Для построения сравнительных графиков и оценки количественных характеристик уединенных волн были взяты числовые значения модулей упругости полистирола (ПС) из [13] и органического стекла (полиметилметакрилата, ПММА) из [14], табл. 1. Значение амплитуды солитона сжатия выбрано равным $A=-2.5 \cdot 10^{-4} \mathrm{~m}$, близким к значению амплитуд солитонов сжатия, распространяющихся в изотропных стержнях из ПС и ПММА [15]. В табл. 2 приведены скорости $c_{0}$ распространения линейных волн, скорости $\mathrm{v}$ нелинейных уединенных волн, значения коэффициентов $\beta$ при нелинейном слагаемом в уравнении Эйлера-Лагранжа, и ширины солитонов на половине высоты FWHM для трех различных волноводов: цилиндрического стержня из ПС радиуса $R=5 \mathrm{~mm}$, такого же стержня из ПММА и двуслойного коаксиального стержня радиуса $R=5 \mathrm{~mm}$, внутренний стержень которого имеет радиус $R_{1}=5 / \sqrt{2} \approx 3.53 \mathrm{~mm}$ и изготовлен из ПС, а внешняя оболочка - из ПММА. Соотношение между $R$ и $R_{1}$ выбрано таким, чтобы площади ПС и ПММА в сечении были одинаковыми. График (20) представлен на Рис. 2 в сравнении с профилями уединенных волн в цилиндрических стержнях из ПС и ПММА.

\section{Заключение}

В настоящей работе исследованы объемные нелинейные продольные волны деформации в двуслойном коаксиальном цилиндрическом стержне. Разработан асимптотический метод вывода уравнений распространения длинных нелинейно-упругих продольных волн деформации в таком волноводе, основанный на асимптотическом удовлетворении условия свободной от напряжений границы, т.е. равенстве нулю компонент тензора напряжений Пиолы-Кирхгоффа $\mathbf{P}$, и на неразрывности напряжений и перемещений на границе раздела слоев. Это позволило получить нелинейное УДД, имеющее частное аналитическое решение в виде уединенных нелинейных волн - объемных солитонов сжатия.

Применение разработанного метода позволило решить задачу о распространении длинных нелинейных упругих продольных волн деформации в двуслойном цилиндрическом стержне с идеальным контактом между слоями. Модель предсказывает формирование единой волны, параметры которой зависят от упругих характеристик материалов обоих слоев. Показано, что в коаксиальном стержне скорость объемного солитона деформации превышает „стержневую“ скорость линейных волн, а параметры солитона лежат между параметрами солитонов в однородных изотропных стержнях тех же размеров, изготовленных из материалов первого, либо второго слоев. Найдены зависимости амплитуды и скорости солитона от радиуса границы раздела материалов слоев, их плотности и упругих характеристик.

\section{Приложения}

Точность вывода нелинейных уравнений и граничных условий краевой задачи напрямую связана с трудоемкими вычислениями. Даже при описанном алгоритме получения соответствующих выражений и коэфффициентов уравнений проведение преобразований остается довольно тяжелой задачей, а без приведения итогов таких вычислений пришлось бы многие результаты решения просто принимать на веру. Поэтому ниже приводятся выражения, необходимые для подтверждения выводов, сделанных в основном тексте и для расчетов в реальных физических задачах.

\section{1. Нелинейные составляющие компонент $P_{4, x x}^{(i)}$ и $\boldsymbol{P}_{4, \varphi \varphi}^{(i)}$ тензора Пиолы-Кирхгоффа}

Нелинейные части выражений $P_{4, x x}^{(i)}$ и $P_{4, \varphi \varphi}^{(i)}$ для тензора Пиолы-Кирхгоффа с учетом найденных выражений для $U_{0}^{(i)}$ и $V_{0}^{(i)}$ и $(13)$

$$
\begin{aligned}
& P_{4, x x N L}^{(1)}=\lambda_{1}\left(\frac{V_{2, N L}^{(1)}}{r}+\partial_{r} V_{2, N L}^{(1)}\right) \\
& +\left(-2 \frac{a_{0}}{d_{0}}\left(\lambda_{1}+2 l_{1}\right)+\frac{a_{0}^{2}}{d_{0}^{2}}\left(4 l_{1}-2 m_{1}+n_{1}+\lambda_{1}\right)\right. \\
& \left.+\left(l_{1}+2 m_{1}+\frac{3}{2} \lambda_{1}+3 \mu_{1}\right)\right)\left(\partial_{x} F\right)^{2},
\end{aligned}
$$




$$
\begin{aligned}
& P_{4, x x N L}^{(2)}=\lambda_{2}\left(\frac{V_{2, N L}^{(2)}}{r}+\partial_{r} V_{2, N L}^{(2)}\right) \\
& +\left(-2 \frac{a_{0}-\sigma_{0}}{d_{0}}\left(\lambda_{2}+2 l_{2}\right)+\frac{a_{0}^{2}-2 a_{0 \sigma_{0}}}{d_{0}^{2}}\right. \\
& \times\left(4 l_{2}-2 m_{2}+n_{2}+\lambda_{2}\right)+\left(l_{2}+2 m_{2}+\frac{3}{2} \lambda_{2}+3 \mu_{2}\right) \\
& \left.+\frac{\sigma_{0}^{2}}{d_{0}^{2}}\left(4 l_{2}-\left(1-\frac{\alpha^{4}}{r^{4}}\right)\left(2 m_{2}-n_{2}\right)+\left(1+\frac{\alpha^{4}}{r^{4}}\right) \lambda_{2}\right)\right) \\
& \times\left(\partial_{x} F\right)^{2}, \\
& \quad P_{4, \varphi \varphi N L}^{(1)}=2 \mu_{1} \frac{V_{2, N L}^{(2)}}{r}+\lambda_{1}\left(\frac{V_{2, N L}^{(1)}}{r}+\partial_{r} V_{2, N L}^{(1)}\right) \\
& \quad+\left(\frac{\lambda_{1}+2 l_{1}}{2}-\frac{a_{0}}{d_{0}}\left(4 l_{1}-2 m_{1}+n_{1}+\lambda_{1}\right)\right. \\
& P_{4, \varphi \varphi N L}^{(2)}=2 \mu_{2} \frac{V_{2, N L}^{(2)}}{r}+\lambda_{2}\left(\frac{V_{2, N L}^{(2)}}{r}+\partial_{r} V_{2, N L}^{(2)}\right) \\
& +\left(\frac{\lambda_{2}+2 l_{1}}{2}-\frac{a_{0}}{d_{0}}\left(4 l_{2}-2 m_{2}+n_{2}+\lambda_{2}\right)\right. \\
& \left.+\frac{a_{0}^{2}}{d_{0}^{2}}\left(4 l_{2}+2 m_{2}+3\left(\lambda_{2}+\mu_{2}\right)\right)\right)\left(\partial_{x} F\right)^{2}, \\
& +\frac{\sigma_{0}}{d_{0}}\left(4 l_{2}-\left(1+\frac{\alpha^{2}}{r^{2}}\right)\left(2 m_{2}-n_{2}\right)+\left(1-\frac{\alpha^{2}}{r^{2}}\right) \lambda_{2}\right) \\
& \frac{\sigma_{0}^{2}}{d_{0}^{2}}\left(2 \lambda_{2}+4 l_{2}+\left(1-\frac{\alpha^{2}}{r^{2}}\right)^{2}\left(\lambda_{2}+2 m_{2}+3 \mu_{2}\right)\right) \\
& \left.+\frac{2 a_{0} \sigma_{0}}{d_{0}^{2}}\left(2 \lambda_{2}+4 l_{2}+\left(1-\frac{\alpha^{2}}{r^{2}}\right)\left(\lambda_{2}+2 m_{2}+3 \mu_{2}\right)\right)\right) \\
& \times\left(\partial_{x} F\right)^{2},
\end{aligned}
$$

\section{2. Степенные разложения функций} перемещений в размерной форме

В размерных переменных формулы (16) выглядят следующим образом:

$$
\begin{gathered}
U^{(1)}=F(x, t)+a_{1} \frac{r^{2}}{2} \partial_{x x} F, r \in\left(0 ; R_{1}\right] \\
U^{(2)}=F(x, t)+\left[\left(a_{1}-\sigma_{1} \frac{2 \ln \alpha}{\alpha^{2}}\right) \frac{r^{2}}{2}+\sigma_{1} P^{2} \ln \frac{r}{R}\right] \partial_{x x} F, \\
r \in\left(R_{1}, R\right], \\
V^{(1)}=-\frac{a_{0}}{d_{0}} r \partial_{x} F+\left[-\frac{a_{2}}{d_{2}} r^{3} \partial_{x x x} F\right], r \in\left(0, R_{1}\right], \\
V^{(2)}=-\left(\frac{a_{0}-\sigma_{0}}{d_{0}} r+\frac{\sigma_{0}}{d_{0}} \frac{\alpha^{2} R^{2}}{r}\right) \partial_{x} F+\left[-\left(\frac{b_{2}}{d_{2}} r^{3}\right.\right. \\
\left.\left.+\frac{c_{2}}{d_{2}} r R^{2}\left(1-\ln \frac{r}{R}\right)\right) \partial_{x x x} F\right], r \in\left(R_{1}, R\right]
\end{gathered}
$$

\section{3. Уравнение распространения длинных нелинейных упругих продольных волн деформации в двуслойном коаксиальном цилиндрическом стержне в размерных переменных}

Уравнение (17) в размерных переменных, важных для расчетов при физических экспериментах, имеет вид

$$
u_{t t}-c_{0}^{2} u_{x x}=\frac{B_{1}}{\bar{\rho}}\left(u^{2}\right)_{x x}+\frac{R^{2}}{\bar{\rho}}\left(B_{2} u_{x x t t}+B_{3} u_{x x x x}\right),
$$

где $c_{0}^{2}=B_{0} / \bar{\rho}, \bar{\rho}=\alpha^{2} \rho_{1}+\left(1-\alpha^{2}\right) \rho_{2}, \alpha-$ безразмерный радиус внутреннего стержня.

В общем случае коэффициенты $B_{i}, i \in[0 ; 3]$, введенные в (17), (23), имеют вид

$$
\begin{aligned}
& B_{0}=\alpha^{2}\left(\lambda_{1}+2 \mu_{1}\right)+\left(1-\alpha^{2}\right)\left(\lambda_{2}+2 \mu_{2}\right) \\
& -4 \frac{a_{0}}{d_{0}}\left(\alpha^{2} \lambda_{1}+\left(1-\alpha^{2}\right) \lambda_{2}\right) \\
& +4 \frac{a_{0}^{2}}{d_{0}^{2}}\left(\alpha^{2}\left(\lambda_{1}+\mu_{1}\right)+\left(1-\alpha^{2}\right)\left(\lambda_{2}+\mu_{2}\right)\right) \\
& +4\left(1-\alpha^{2}\right) \frac{\sigma_{0}}{d_{0}}\left[\lambda_{2}-2 \frac{a_{0}}{d_{0}}\left(\lambda_{2}+\mu_{2}\right)\right. \\
& \left.+\frac{\sigma_{0}}{d_{0}}\left(\lambda_{2}+\left(1+\alpha^{2}\right) \mu_{2}\right)\right], \\
& B_{1}=\alpha^{2}\left(3 \lambda_{1}+6 \mu_{1}+2 l_{1}+4 m_{1}\right) \\
& +\left(1-\alpha^{2}\right)\left(3 \lambda_{2}+6 \mu_{2}+2 l_{2}+4 m_{2}\right)-6 \frac{a_{0}}{d_{0}}\left(\alpha^{2}\left(\lambda_{1}+2 l_{1}\right)\right. \\
& \left.+\left(1-\alpha^{2}\right)\left(\lambda_{2}+2 l_{2}\right)\right)+6 \frac{a_{0}^{2}}{d_{0}^{2}}\left(\alpha^{2}\left(\lambda_{1}+4 l_{1}-2 m_{1}+n_{1}\right)\right. \\
& \left.+\left(1-\alpha^{2}\right)\left(\lambda_{2}+4 l_{2}-2 m_{2}+n_{2}\right)\right) \\
& -4 \frac{a_{0}^{3}}{d_{0}^{3}}\left(\alpha^{2}\left(3 \lambda_{1}+3 \mu_{1}+4 l_{1}+2 m_{1}\right)+\left(1-\alpha^{2}\right)\right. \\
& \left.\times\left(3 \lambda_{2}+3 \mu_{2}+4 l_{2}+2 m_{2}\right)\right) \\
& +6 \frac{\sigma_{0}}{d_{0}}\left(1-\alpha^{2}\right)\left[\lambda_{2}+2 l_{2}-2 \frac{a_{0}}{d_{0}}\left(\lambda_{2}+4 l_{2}-2 m_{2}+n_{2}\right)\right. \\
& \left.+2 \frac{a_{0}^{2}}{d_{0}^{2}}\left(3 \lambda_{2}+3 \mu_{2}+4 l_{2}+2 m_{2}\right)\right] \\
& +6 \frac{\sigma_{0}^{2}}{d_{0}^{2}}\left(1-\alpha^{2}\right)\left[\left(1+\alpha^{2}\right) \lambda_{2}+4 l_{2}+\left(1-\alpha^{2}\right)\left(-2 m_{2}+n_{2}\right)\right. \\
& -2 \frac{a_{0}}{d_{0}}\left(\left(\alpha^{2}+3\right) \lambda_{2}+4 l_{2}+\left(1+\alpha^{2}\right)\left(3 \mu_{2}+2 m_{2}\right)\right) \\
& +4 \frac{\sigma_{0}^{3}}{d_{0}^{3}}\left(1-\alpha^{2}\right)\left[3\left(1+\alpha^{2}\right) \lambda_{2}+3\left(1+3 \alpha^{2}\right) \mu_{2}\right) \\
& \left.+4 l_{2}+2\left(1+3 \alpha^{2}\right) m_{2}\right],
\end{aligned}
$$

Журнал технической фризики, 2017, том 87, вып. 12 


$$
\begin{aligned}
& B_{2}=\left(\frac{a_{0}^{2}}{2 d_{0}^{2}}-\frac{a_{1}}{2}\right)\left(\alpha^{4} \rho_{1}+\left(1-\alpha^{4}\right) \rho_{2}\right) \\
& -\frac{a_{0} \sigma_{0}}{d_{0}^{2}} \rho_{2}\left(1-\alpha^{2}\right)^{2} \\
& +\frac{\sigma_{0}^{2}}{2 d_{0}^{2}} \rho_{2}\left[\left(1-\alpha^{2}\right)\left(1-3 \alpha^{2}\right)-4 \alpha^{4} \ln \alpha\right] \\
& +\sigma_{1} \rho_{2}\left[1-\alpha^{2}+\frac{1+\alpha^{4}}{\alpha^{2}} \ln a\right] \\
& B_{3}=\left(1-\frac{2 a_{0}}{d_{0}}\right)\left(\frac{\alpha_{1}}{2}\left(\alpha^{4} \lambda_{1}+\left(1-\alpha^{4}\right) \lambda_{2}\right)-\frac{4}{d_{2}}\left(\alpha^{4} a_{2} \lambda_{1}\right.\right. \\
& \left.\left.+\left(1-\alpha^{4}\right) b_{2} \lambda_{2}\right)\right)+\left(a_{1}\left(1+\frac{a_{0}}{d_{0}}\right)-\frac{a_{1}^{2}}{2}-\frac{a_{0}^{2}}{2 d_{0}^{2}}\right) \\
& \times\left(\alpha^{4} \mu_{1}+\left(1-\alpha^{4}\right) \mu_{2}\right)+\frac{8 a_{0}}{d_{0} d_{2}}\left(\alpha^{4} a_{2} \mu_{1}+\left(1-\alpha^{4}\right) b_{2} \mu_{2}\right) \\
& +\frac{\sigma_{0}}{d_{0}}\left(1-\alpha^{4}\right)\left[\left(1+\alpha^{2}\right)\left(a_{1}-\frac{8 b_{2}}{d_{2}}\right) \lambda_{2}\right. \\
& \left.-\left(8\left(1+2 \alpha^{2}\right) \frac{b_{2}}{d_{2}}+\left(1-\alpha^{2}\right)\left(a_{1}-\frac{a_{0}}{d_{0}}\right)\right) \mu_{2}\right] \\
& +\frac{\sigma_{1}}{d_{0}}\left[\left(1-\alpha^{2}+\frac{1+\alpha^{4}}{\alpha^{2}} \ln \alpha\right)\left(2 a_{0}-d_{0}\right) \lambda_{2}\right. \\
& +2\left(1-\alpha^{2}-\frac{1-\alpha^{4}}{\alpha^{2}} \ln \alpha\right)\left(a_{0}-\left(1+a_{1}\right) d_{0}\right) \mu_{2} \\
& \left.-4 \mu_{2} d_{0} \frac{\ln \alpha}{\alpha^{2}}\right]-\frac{8 \sigma_{0} c_{2}}{d_{0} d_{2}}\left[\left(1-\alpha^{2}+\alpha^{2} \ln \alpha\right) \lambda_{2}\right. \\
& \left.+\left(1-\alpha^{2}+2 \alpha^{2} \ln \alpha\right) \mu_{2}\right]-\frac{2 \sigma_{0} \sigma_{1}}{d_{0}}\left[\left(1-\alpha^{2}\right.\right. \\
& \left.\left.+\frac{1+\alpha^{4}}{\alpha^{2}} \ln \alpha\right) \lambda_{2}+\left(1-\alpha^{2}-\frac{1-2 \alpha^{2}-\alpha^{4}}{\alpha^{2}} \ln \alpha\right) \mu_{2}\right] \\
& \left.-\frac{\sigma_{0}^{2}}{2 d_{0}^{2}}\left(1-4 \alpha^{2}\right)+3 \alpha^{4}-4 \alpha^{4} \ln \alpha\right) \mu_{2} \\
& -\sigma_{1}^{2}\left(2-\frac{4}{\alpha^{2}}-\left(2-\frac{2}{\alpha^{4}}\right) \ln \alpha\right) \mu_{2}
\end{aligned}
$$

\section{Список литературы}

[1] Samsonov A.M., Dreiden G.V., Semenova I.V. // Proc. Inst. Mech. Eng. Part C. J. Mech. Engng. Sci. 2008. Vol. 22. N 10. P1975-1980,

[2] Dreiden G.V., Khusnutdinova K.R., Samsonov A.M., Semenova I.V. // J. Appl. Phys. 2008. Vol. 104. N 8. P. 086106.

[3] Dreiden G.V., Khusnutdinova K.R., Samsonov A.M., Semenova I.V. // J. Appl. Phys. 2010. Vol. 107. N 3. P. 034909.

[4] Dreiden G.V., Khusnutdinova K.R., Samsonov A.M., Semenova I.V. // J. Appl. Phys. 2012. Vol. 112. N 6. P. 063516.
[15] Sertakov I., Engelbrecht J., Janno J. // Int. J. Solids Struct. Vol. 49. 2014. P. 3381-3387.

[6] Khusnutdinova K.R., Samsonov A.M. // Phys. Rev. E. 2008. Vol. 7. P. 066603.

[7] Khusnutdinova K.R., Tranter M.R. // Proc. R. Soc. A. 2015. Vol. 471. P. 20150584.

[8] Moulana M., Nariboli G.A. // Acta Mech. 1976. Vol. 24. P. 1324.

[9] Nariboli G.A. // ZAMM. 1969. Vol. 49. P. 525-531.

[10] Samsonov A.M. Strain solitions in solids and how to construct them. Chapman \& Hall/CRC, 2001. 248 p.

[11] Porubov A.V., Andrianov I.V., Danishevs'kyy V.V. // Int. J. Solids Struct. 2012. Vol. 49. P. 3381-3387.

[12] Murnaghan F.D. Finit deformation of an elastic solid. Chapman \& Hall, 1951. 140 p.

[13] Hughes D.S., Kelly J.L. // Phys. Rev. 1953. Vol. 92. N 5. P. 1145-1149.

[14] Semenova I.V., Dreiden G.V., Samsonov A.M. Dynamic Behavior of Materials, Vol. 1: Proc. 2010 Annual Conference on Experimental and Applied Mechanics. / Ed. by. T. Proulx. N. Y. : Springer, 2011. P. 261-267.

[15] Samsonov A.M., Semenova I.V., Belashov A.V. // Wave Motion. 2016. 10.1016/j.wavemoti.2016.06.006. 